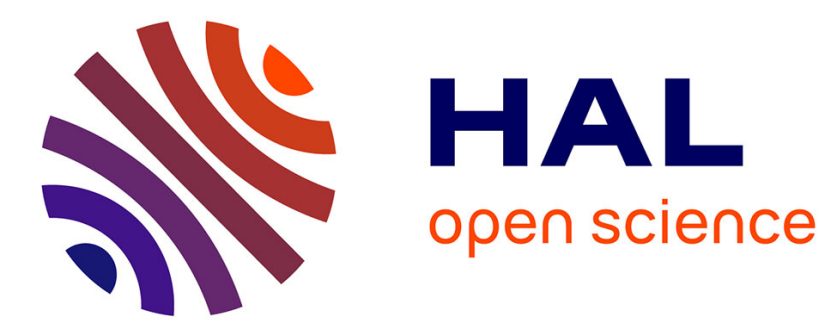

\title{
Dysfonctions sexuelles après traumatisme crânien : revue systématique de la littérature
}

\author{
Hélène Robert, Bertrand Pichon, R. Haddad
}

\section{To cite this version:}

Hélène Robert, Bertrand Pichon, R. Haddad. Dysfonctions sexuelles après traumatisme crânien : revue systématique de la littérature. Progrès en Urologie, 2019, 29 (11), pp.529-543. 10.1016/j.purol.2019.08.002 . hal-02974768

\section{HAL Id: hal-02974768 https://hal.sorbonne-universite.fr/hal-02974768}

Submitted on 4 Nov 2020

HAL is a multi-disciplinary open access archive for the deposit and dissemination of scientific research documents, whether they are published or not. The documents may come from teaching and research institutions in France or abroad, or from public or private research centers.
L'archive ouverte pluridisciplinaire HAL, est destinée au dépôt et à la diffusion de documents scientifiques de niveau recherche, publiés ou non, émanant des établissements d'enseignement et de recherche français ou étrangers, des laboratoires publics ou privés. 


\title{
Sexual Dysfunctions After Traumatic Brain Injury: Systematic Review Of The Literature
}

\section{Robert H, Pichon B, Haddad R}

\begin{abstract}
Background: Sexual dysfunctions (SD) are common after a traumatic brain injury (TBI) but remain underrated in clinical practice, yet it is a crucial aspect of the person with consequences for the relationship with the other, psychological well-being and quality of life.

Objective: To determine, through a systematic literature review, the epidemiology, assessment tools and treatment of SD in the TBI population.
\end{abstract}

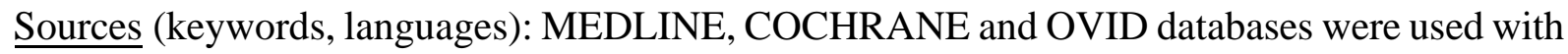
specific keywords (MeSH), combined with Boolean operators: "sexual dysfunction", "sexuality", "erectile dysfunction" and "traumatic brain injury".

Study selection: Only studies published in French or English, and with full-text available, have been included. Articles have been independently reviewed and extracted.

Results: Of the 199 articles reviewed after exclusion of duplicates, 86 articles were reviewed in their full text. A total of 40 studies were included in the final analysis. After TBI, $6 \%$ to $83 \%$ of patients report SD: decreased frequency of sexual intercourse, desire and/or arousal, erectile dysfunction, difficulties with orgasm, inappropriate sexual behaviour. There is no consensus method for evaluating SD in this population, with 16 tools identified. Among them, only two questionnaires were validated in this population, the Brain Injury Questionnary of Sexuality - not validated in French - and the Overt Behavior Scale, the latter being intended for the evaluation of sexual behaviour disorders. Several factors are significantly and positively associated with SD: age, severity of TBI, depression, anxiety, and fatigue. Others are negatively associated: time since injury, perceived physical health status and social participation. There is little data on the treatment of SD outside of case studies. 
Limits: Quantitative analysis could not be performed due to differences in the studies included in their design, evaluation tools, choice of TBI severity criteria, and post-TBI timeframes. Four unavailable articles could not be consulted.

Conclusion: SD are common after TBI but remain poorly evaluated in clinical practice, despite their impact on patients and their partners. Their evaluation and treatment should be part of the overall management of patients after TBI. Nevertheless, there is currently no validated tool in French to evaluate these SD, nor are there any guidelines on their treatment.

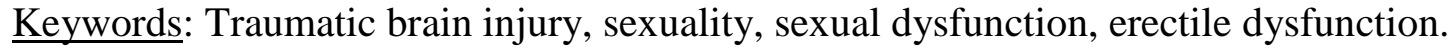




\section{INTRODUCTION}

L’OMS définit la santé sexuelle comme « un état de bien-être physique, mental et social dans le domaine de la sexualité » (1). Selon Thorn-Gray et Kern, la sexualité est définie comme « la communication verbale, visuelle, tactile et olfactive qui exprime l'amour et l'intimité entre deux personnes » (2). Elle englobe des dimensions biologiques, physiques, culturelles, psychosociales et comportementales, chacune pouvant être perturbée par diverses pathologies, dont les maladies neurologiques. Parmi ces causes neurologiques, les traumatismes crâniens (TC), représentent une cause majeure de décès et de handicaps pour la population des adultes

jeunes (3). En effet, l'incidence annuelle des TC hospitalisés est estimée entre 150 et 300 pour 100000 habitants en France. Les hommes sont plus touchés que les femmes (3/1), principalement les adultes jeunes. Les lésions associées sont fréquentes, sources de séquelles, ce d'autant que le TC est grave. Cette gravité peut être déterminée par le Glasgow Coma Scale (GCS) et la durée de l'amnésie post-traumatique (4). Après un TC, les patients et/ou leur famille peuvent rapporter des troubles cognitifs, des modifications comportementales et des déficiences physiques (5). Parmi ces modifications, les dysfonctions sexuelles (DS), communes dans la population générale (6), sont plus fréquentes après un TC. En dépit de leur impact sur la relation à l'autre, le bien-être psychologique et la qualité de vie (5), elles ne sont décrites que depuis 1976 (7) et les DS restent peu évaluées en pratique clinique (8). L’objectif de cette revue systématique de la littérature est de déterminer, dans la population des TC, l'épidémiologie, les outils d'évaluation et le traitement des dysfonctions sexuelles.

\section{MATERIEL ET METHODES}

Cette revue systématique de la littérature a été menée en accord avec les recommandations PRISMA (Preferred Reporting Items for Systematic Reviews and Meta-Analyses)(9).

\section{Critères d'éligibilité}




\section{Types d'études}

Les études originales, y compris les essais contrôlés randomisés, les études non- randomisées, de cohorte, cas-témoins, transversales et les séries de cas, ont été inclues. Les articles de synthèse et les méta-analyses n'ont pas été inclus.

\section{Population}

Seules les études impliquant des adultes ont été sélectionnées. Les sujets inclus devaient être les patients ayant un TC ou leur partenaire. Aucune limite de sexe, d'appartenance ethnique, de milieu, de localisation, sévérité ou d’évolution du TC n'a été appliquée. Concernant les DS, elles pouvaient concerner les altérations physiologiques (désir, excitation, lubrification, érection, orgasme, éjaculation, douleur), psychologiques de la sexualité ou les troubles du comportement sexuel (impulsivité, paraphilies, etc.). Les DS pouvaient être évaluées par des questionnaires spécifiques ou non.

\section{Données d'intérêt}

Les articles devaient inclure des données sur l'épidémiologie, les outils d'évaluation et le traitement des DS chez les patients avec TC.

\section{Stratégie de recherche et sources d'information}

Les bases de données MEDLINE, COCHRANE et OVID ont été utilisées avec des mots clés spécifiques (MeSH), combinés avec des opérateurs booléens : « sexual dysfunction », « sexuality », " erectile dysfunction » et « traumatic brain injury ». La recherche s'est terminée en mai 2019 et aucune date limite n'a été fixée. Seules les études publiées en français ou en anglais ont été inclues. Les références citées dans les revues systématiques ou les méta-analyses ont été recherchées. Une fois les articles clés identifiés, des recherches supplémentaires ont été effectuées sur PubMed à l'aide de la fonction de recherche "Related Articles". Une recherche 
additionnelle concernant les recommandations pour la prise en charge de ces patients a été réalisée sur les site des sociétés savantes d’urologie, de neurologie et de sexologie comme l'American Urological Association, l'European Urological Association, l'Association Française d’Urologie, l’European Academy of Neurology, l'International Society for Sexual Medicine et la Bristish Society for Sexual Medicine.

\section{Collecte et analyse des données}

\section{Sélection des études}

Les auteurs de la revue (HR et $\mathrm{RH}$ ) ont examiné de façon indépendante les titres et les résumés issus de la recherche bibliographique. Le texte intégral des articles a été obtenu pour tous les titres qui semblaient répondre aux critères d'inclusion ou pour lesquels il $\mathrm{y}$ avait des incertitudes. Les auteurs de l'étude ont ensuite passé en revue l'ensemble des articles et déterminé de façon indépendante s'ils répondaient aux critères d'inclusion. Les désaccords ont été résolus par la discussion. Les raisons d'exclusion des articles ont été consignées. La Figure 1 résume ce processus de sélection.

\section{Extraction des données}

Les données ont été extraites indépendamment (HR et RH) à l'aide d'un tableur standard, qui recueillait les informations sur le type d'étude, la population, la prévalence des DS, les facteurs associés aux DS, leur évaluation et leur traitement. Un résumé est présenté dans la section des résultats (Tableaux 1, 2, 3 et 4 respectivement).

\section{RESULTATS}

\section{Sélection des études}


Un diagramme résumant le processus de recherche et de sélection des articles est présenté par la Figure 1. Sur les 199 articles examinés après exclusion des doublons, 86 articles ont été étudiés dans leur texte intégral. Au total, 40 études ont été inclues dans l'analyse finale. Parmi elles, de façon non exclusive, 16 évaluaient la prévalence des DS chez les patients avec TC (3,6,10-23), 9 chez les couples ou les partenaires (24-32), 23 les facteurs associés aux DS (3,6,10-23,25,27,30,31,33-38) et seulement 4 les traitements des DS (39-42).

\section{Prévalence des DS chez les patients avec un TC}

Au total, 25 études estiment la prévalence de ces troubles chez les patients avec TC ou leur partenaire, parmi lesquelles 13 études transversales (10-14,17,19,21,26-29,32), 8 cas-témoin (3,6,15,20,22-24,30), 3 de cohorte $(16,25,31)$ et une étude de cas (18) (Tableau 1). La prévalence des DS varie de 6\% à 83\% chez les TC modérés à sévères : diminution du désir et/ou de l'excitation, de la fréquence des rapports sexuels (RS), dysorgasmie et dysfonction érectile (DE), allant du plus au moins fréquemment rapporté. Seules 3 études rapportent la survenue de comportements sexuels inappropriés après un TC $(16,18,21)$.

\section{En fonction de la gravité du TC}

Seule une étude porte sur des TC légers (perte de connaissance initiale $<15 \mathrm{~min}$ ), et rapporte 58\% de DS dont 53\% de diminution du désir et 42\% de DE (13). Trois études $(17,21,30)$ portent uniquement sur des TC sévères (GCS < 9), dont une sur les comportement sexuels inappropriés (21), dont la prévalence est estimée à 8,9\%, avec dans la majorité des cas des paroles à caractère sexuel. Si Quintard et al. rapportent une insatisfaction sexuelle chez 68\% des patients (17), sans plus de précisions sur le type de DS, Bivona et al. relatent une diminution du désir et de la fréquence des RS chez les patients et leur partenaire (30). Les autres études incluent tous les degrés de sévérité de TC, avec une majorité de TC modérés à sévères. 


\section{En fonction du délai d'évolution du TC}

Une seule étude évalue les DS à la phase subaiguë (6 semaines) du TC (14), les autres études étant réalisées à 1 an ou plus du TC : 3 à 1 an (19,31,35), 4 à plus d'1 an (3,6,8,10), 7 à plus de 2 ans $(11,13,15,20,30,32,34)$ et 2 à plus de 5 ans $(12,17)$. A 6 semaines du TC, seulement14\% des patients rapportent une DS, principalement une DE pour ceux en couple et une diminution du désir pour les célibataires, mais il est possible qu'à cette phase les patients aient d'autres préoccupations (14). A un an ou plus du TC : 24\% (19) à 86\% (15) des patients signalent une diminution du désir, 47\% (12) à 62\% (19,31) une diminution de la fréquence des RS et environ 29 à 40\% ont une dysorgasmie $(12,19)$. Une seule étude a rapporté de manière prospective l'évolution des DS entre 6 et 12 mois d’un TC modéré à sévère (33). Hormis l’excitation qui s’améliore, il n’existe pas de différence significative pour la satisfaction sexuelle, les fantasmes, les expériences sexuelles ou l’orgasme.

\section{En fonction du sexe}

Onze études (3,6,11,12,16,17,19-22,33) portent sur les DS chez les femmes avec un TC, mais sept études ne font pas de distinction hommes-femmes $(3,11,12,17,20,22,33)$ et une seule a inclus exclusivement des femmes (6). La prévalence globale de DS allait de 35 (19) à 83\% (6), avec, par ordre de fréquence : diminution du désir ou de l’excitation, trouble de la lubrification, diminution de la satisfaction sexuelle, dysorgasmie et dyspareunie (6). Cette prévalence de DS n’était pas significativement différente chez les femmes sans TC dans une étude (24). Dix-sept études portent sur les hommes $(10,13-15,23,30)$ dont 6 de manière exclusive $(3,10-23,25-32)$. La prévalence globale allait de 58\% (13) à 100\% (15), avec, par ordre de fréquence : de 62\% (10) à 86\% (15) une diminution de la fréquence des RS, 24,2\% (19) à 57\% (10) une DE, plus d'un tiers une dysorgasmie $(10,15)$. Yang et al. retrouvent une incidence de DE significativement plus importante dans la population TC, que dans la population générale (23). 
Deux études ont retrouvé une insatisfaction sexuelle plus importante chez les hommes que chez les femmes après un TC $(17,19)$. Les hommes ont tendance à avoir plus de comportements sexuels inappropriés que les femmes $(16,21)$.

\section{Les DS chez les partenaires}

La prévalence des DS chez les partenaires varie de 9\% (27) à 20 \% (29), avec le même éventail de troubles que ceux constatés chez les patients : diminution du désir, de l'excitation, DE, dysorgasmie. Si dans certaines études les partenaires rapportent moins de DS (9\%) que les patients (50\%) (27) et notamment moins de trouble de la lubrification (32), d'autres allèguent le contraire, comme dans le travail de Garden et al., où 64\% des partenaires femmes rapportent une dysorgasmie après le TC de leur conjoint, contre 50\% des femmes ayant eu un TC (26). Les couples signalent tous une diminution de la fréquence des RS, avec jusqu'à 78,6\% des couples n’ayant plus de RS depuis le TC (28).

\section{Facteurs associés aux DS}

Parmi les 23 études évaluant les facteurs associés aux DS, seules 9 utilisent des modèles multivariés pour déterminer l'indépendance de cette association(6,12,17,22,23,31,34,35,37) (Tableau 2). Ainsi, l'état de santé physique perçu (12), la participation sociale (35), l’âge $(6,17,22,31,34,37)$, la gravité du TC $(17,23,35)$, le délai d’évolution $(6,34)$, la dépression (34,37), l'anxiété (37) et la fatigue (22) sont des facteurs indépendants de DS. Le sens de l'association entre sexe et DS est variable en fonction de ces études, avec parfois le sexe masculin (17) ou féminin $(22,35)$ retrouvés comme facteur de risque de DS. Seule une étude prend en compte la iatrogénie, et il faut noter l'absence d'association entre prise d'antidépresseur et DS quand la relation est ajustée sur la dépression (34). Concernant les facteurs associés aux 
DS chez le partenaire, sont retrouvés en analyse univariée : l’âge et le fonctionnement sexuel du patient (31) et l’anxiété et la dépression (27) chez le partenaire.

\section{Outils d'évaluation des DS}

Un total de 16 outils standardisés a été utilisé pour évaluer les DS dans les études inclues (Tableau 3). Parmi ces méthodes d'évaluation, le Brain Injury Questionnary of Sexuality (BIQS), utilisé dans 3 études (20,31,34) a été validé dans une population de TC. Il est utilisable chez les hommes et les femmes, mais il n’est pas normé et n’est pas validé en Français. Le BIQS est un auto-questionnaire se composant de 3 items: Fonctionnement sexuel (8 questions), Qualité de la Relation et Estime de Soi (5 questions), Humeur (2 questions). C’est une version améliorée du Sexual Questionnaire (Cronbach $\alpha=0,92)$, adapté du Psychosexual Assesment Questionnaire (PAQ), qui s’il n’est pas validé, a été créé pour une population de TC $(10,12)$. Le BIQS a de bonnes propriétés psychométriques avec une bonne cohérence interne (Cronbach $\alpha=0$,81-0,94) et une bonne validité convergente comparée au Derogatis Interview for Sexual Functioning-Self Report Version (DISF-SR). Un autre questionnaire, l'Overt Behavior Scale (OBS), a été validé dans cette population (43) , mais s’intéresse aux comportements sexuels inappropriés et est utilisé dans une seule étude (21). Les autres questionnaires n’ont pas été validés dans une population de TC, mais le Sexual adjustement Questionnaire (SAQ) et le Sexual Interest and Satisfaction (SIS) ont été validés dans une population de médullo-lésés. Quand un questionnaire standardisé n’était pas utilisé, les auteurs avaient fait le choix d’entretiens non structurés, semi-structurés, de questionnaires non publiés ou d'utilisation de codes de la CIM-10 $(13,22-25,28,29,44)$.

\section{Traitement des DS}

Il n’existe aucune recommandation des différentes sociétés savantes concernant le traitement des DS chez les patients avec TC. Les recommandations spécifiques de neuro-sexologie 
n’abordent que les aspects cliniques et d'évaluation de ces patients (45). Dans un article de revue, Denys et al. donnent des recommandations globales, comme la nécessité de traiter les troubles vésico-sphinctériens et ano-rectaux avant d'envisager un traitement des DS (46). Quelques articles (Tableau 4) évaluent de façon spécifique des traitements des DS chez les TC, mais il s’agit pour la majorité de séries de cas (39-41). Une étude non randomisée rapporte les résultats de l’ajout de Miansérine chez 17 patients avec une DS sous inhibiteur de la recapture de la sérotonine, consistant en une éjaculation retardée pour 70,5\% d'entre eux. A 2 mois, tous les patients avec une éjaculation retardée avaient une normalisation de leur éjaculation. Enfin, même si des essais thérapeutiques manquent dans cette indication et cette population, deux équipes ont développé des programmes d’éducation sur cette thématique : le programme «You and Me » (47), évalué en 2004 auprès de 37 professionnels et 12 patients après un TC et celui de Sander et al. (48). Le $1^{\text {er }}$ se compose de 15 modules abordant des thèmes différents autour de la sexualité : définition de la sexualité, des droits sexuels et des comportements inappropriés, les DS sont abordées, ainsi que la contraception, la grossesse et les infections sexuellement transmissibles. Celui de Sander et al. définit les différentes DS, en explique les causes et donne quelques conseils : en parler avec son médecin, faire un examen gynécologique/urologique, suivre une prise en charge avec un psychologue et/ou un sexologue, planifier les rapports sexuels quand le patient est le moins fatigué, choisir des positions non douloureuses, améliorer les relations sociales. Enfin, il comporte une partie «prévention » et rappelle la nécessité d'avoir une contraception et des RS protégés.

\section{DISCUSSION}

Les DS sont fréquentes après un TC, que ce soit chez le patient ou son partenaire. Ces dysfonctions concernent les aspects physiologiques - désir, excitation, lubrification, érection, orgasme, éjaculation - et psychologiques de la sexualité, mais également les troubles du 
comportement sexuel. Dans cette première revue systématique de la littérature sur le sujet, vingt-cinq études ont évalué la prévalence et vingt-trois les facteurs associés aux DS dans cette population. Il est difficile de comparer toutes ces études, en raison des différences dans leur design, leurs outils d'évaluation, leurs choix de critères de gravité du TC, leurs délais post-TC. De plus, seul un petit nombre d'études utilise un groupe contrôle permettant d'attribuer au TC la différence de prévalence de DS observée. Enfin, 4 articles non disponibles n’ont pas pu être consultés.

Concernant l'évaluation des DS, il n'existe pas de méthode consensuelle dans cette population, si bien que 16 outils différents ont été recensés. Parmi eux, seuls deux questionnaires ont été spécifiquement validés dans une population de TC, le BIQS - non validé en langue française et l’OBS, ce dernier étant destiné à l'évaluation des troubles du comportement sexuel. Il s’agissait pour la majorité d'auto-questionnaires, avec de très probables biais subjectif ou de rappel, avec tendance à embellir la vie d'avant (5), ce qui est constaté dans l'étude de Bivona et al. : les patients TC et leurs partenaires avaient une meilleure sexualité avant comparée à celle des contrôles (30). Le couple est donc impacté par le TC et les partenaires rapportent les mêmes types de DS que les patients. Les DS dans le couple pourraient être liées à la détérioration de la relation et à un manque de communication $(34,35)$. Les partenaires rapportent également comme raisons aux DS le stress, leur propre fatigue, le changement de comportement et/ou la fatigue du patient et le sentiment d'être dans une relation de « soignant/aidant » plutôt que dans une relation de couple. D’après une étude qualitative, les facteurs favorisant une meilleure intimité étaient : une plus longue relation avant le TC, passer du temps en couple et avoir un soutien extérieur (famille, amis) (44).

Au-delà de cette analyse qualitative, plusieurs facteurs sont associés de façon significative et positive aux DS : l’âge, la gravité du TC, la dépression, l’anxiété et la fatigue. D’autres sont associés négativement : le délai d'évolution, l'état de santé physique perçu et la participation 
sociale. D’autres facteurs sont rapportés comme associés aux DS, mais étudiés uniquement en analyse univariée et donc assujettis à l'existence de facteurs de confusion non pris en compte.

Tout comme pour l'évaluation des DS, concernant le traitement, le consensus fait encore défaut : il n’existe pas de recommandations spécifiques émanant de sociétés savantes et les seules études disponibles dans cette population sont pour la majorité des séries de cas. En effet, la majorité des articles s’intéresse plutôt aux causes potentielles des DS chez les TC, et au bilan clinique et paraclinique à réaliser (49). Il existe à l’évidence une discordance entre les croyances des professionnels vis-à-vis des traitements et ce qu'ils proposent effectivement. En effet, dans une étude menée sur l'évaluation des pratiques de 324 professionnels à travers le monde, 54\% pensent que les médicaments, l’éducation, la psychothérapie et/ou la sexothérapie font parties intégrantes du panel thérapeutique. La prise en charge avec un sexologue était également rapportée comme particulièrement intéressante pour aider à améliorer l’image du corps, la prise en charge de la dyspareunie et pour apprendre à être à l'aise avec sa sexualité. Néanmoins, la majorité des centres de rééducation n’ont pas de protocole et/ou de recommandations sur la prise en charge des troubles sexuels des TC : seuls 21,8\% des professionnels utilisent un protocole (50).

Pourtant, les troubles sexuels sont une source d’insatisfaction majeure après un TC sévère (46). Selon les études, 67\% (10) à 79\% (15) des patients déclarent une perte d'estime de soi en lien avec ces difficultés, et 71\% disent être déprimés (15). Même si l'évaluation des DS devrait faire partie intégrale de l'évaluation neurologique (51), le praticien se préoccupe le plus souvent de la sexualité lorsqu’elle est inappropriée (15). Cela ne correspond pas à la demande des patients avec un TC, qui pour 68\% d'entre eux souhaitent aborder la question de la sexualité lors de la prochaine visite avec le médecin (19). Néanmoins, 22\% ne parleront des troubles sexuels que si le médecin initie la question et seulement $10 \%$ ne veulent pas aborder la question (19). Les professionnels semblent également gênés (9,3\%) ou incompétents (29,3\%) pour aborder le sujet 
et seulement 36\% l'abordent dont 23,6\% de manière systématique dans le cadre du suivi $(8,50)$. Lors de l'évaluation d'un programme spécifique de Réadaptation réalisé en Hôpital de Jour du service de MPR de la Pitié-Salpêtrière, pour des patients après lésions cérébrales acquises, le point négatif le plus rapporté était que la sexualité n’était pas abordée dans ce programme (52).

\section{CONCLUSION}

Les dysfonctions sexuelles sont fréquentes après un traumatisme crânien mais restent peu évaluées en pratique clinique. Pourtant, il s’agit d’un aspect crucial de la personne ayant un impact sur la relation avec le partenaire, l'état thymique et la qualité de vie. L'évaluation et la prise en charge des dysfonctions sexuelles devraient faire partie de la prise en charge globale des patients après un TC. Néanmoins, il n’existe pas actuellement d’outil validé en français pour évaluer ces DS, ni de recommandations sur leur traitement. 


\section{REFERENCES}

1. OMS | Santé sexuelle [Internet]. WHO. [cité 22 mai 2019]. Disponible sur: https://www.who.int/topics/sexual_health/fr/

2. Thorn-Gray BE, Kern LH. Sexual dysfunction associated with physical disability: a treatment guide for the rehabilitation practitioner. Rehabil Lit. juin 1983;44(5-6):138-44.

3. Ponsford J. Sexual changes associated with traumatic brain injury. Neuropsychol Rehabil. 1 janv 2003;13(1-2):275-89.

4. Masson F. Épidémiologie des traumatismes crâniens graves. Ann Fr Anesth Réanimation. 1 avr 2000;19(4):261-9.

5. Latella D, Maggio MG, De Luca R, Maresca G, Piazzitta D, Sciarrone F, et al. Changes in sexual functioning following traumatic brain injury: An overview on a neglected issue. J Clin Neurosci. 1 déc 2018;58:1-6.

6. Strizzi J, Olabarrieta Landa L, Pappadis M, Olivera SL, Valdivia Tangarife ER, Fernandez Agis I, et al. Sexual Functioning, Desire, and Satisfaction in Women with TBI and Healthy Controls. Behav Neurol. 2015;2015:247479.

7. Bond MR. Assessment of the psychosocial outcome of severe head injury. Acta Neurochir (Wien). 1976;34(1-4):57-70.

8. Katz S, Aloni R. Sexual dysfunction of persons after traumatic brain injury: perceptions of professionals. Int J Rehabil Res Int Z Rehabil Rev Int Rech Readaptation. mars 1999;22(1):45-53.

9. Moher D, Liberati A, Tetzlaff J, Altman DG, PRISMA Group. Preferred reporting items for systematic reviews and meta-analyses: the PRISMA statement. PLoS Med. 21 juill 2009;6(7):e1000097.

10. Kreutzer JS, Zasler ND. Psychosexual consequences of traumatic brain injury: methodology and preliminary findings. Brain Inj. juin 1989;3(2):177-86.

11. Sandel ME, Williams KS, Dellapietra L, Derogatis LR. Sexual functioning following traumatic brain injury. Brain Inj. oct 1996;10(10):719-28.

12. Kreuter M, Dahllöf AG, Gudjonsson G, Sullivan M, Siösteen A. Brain Inj. mai 1998;12(5):349-68.

13. Kosteljanetz M, Jensen TS, Nørgård B, Lunde I, Jensen PB, Johnsen SG. Sexual and hypothalamic dysfunction in the postconcussional syndrome. Acta Neurol Scand. mars 1981;63(3):169-80.

14. Aloni A, Keren O, Cohen M, Rosentul N, Romm M, Groswasser Z. Incidence of sexual dysfunction in TBI patients during the early post-traumatic in-patient rehabilitation phase. Brain Inj. févr 1999;13(2):89-97.

15. Crowe SF, Ponsford J. The role of imagery in sexual arousal disturbances in the male traumatically brain injured individual. Brain Inj. mai 1999;13(5):347-54. 
16. Simpson G, Blaszczynski A, Hodgkinson A. Sex offending as a psychosocial sequela of traumatic brain injury. J Head Trauma Rehabil. déc 1999;14(6):567-80.

17. Quintard B, Croze P, Mazaux JM, Rouxel L, Joseph PA, Richer E, et al. [Life satisfaction and psychosocial outcome in severe traumatic brain injuries in Aquitaine]. Ann Readaptation Med Phys Rev Sci Soc Francaise Reeducation Fonct Readaptation Med Phys. nov 2002;45(8):456-65.

18. Bianchi-Demicheli F, Rollini C, Lovblad K, Ortigue S. "Sleeping Beauty paraphilia »: deviant desire in the context of bodily self-image disturbance in a patient with a fronto-parietal traumatic brain injury. Med Sci Monit Int Med J Exp Clin Res. févr 2010;16(2):CS15-17.

19. Sander AM, Maestas KL, Pappadis MR, Sherer M, Hammond FM, Hanks R. Sexual Functioning 1 Year After Traumatic Brain Injury: Findings From a Prospective Traumatic Brain Injury Model Systems Collaborative Study. Arch Phys Med Rehabil. 1 août 2012;93(8):1331-7.

20. Downing MG, Stolwyk R, Ponsford JL. Sexual changes in individuals with traumatic brain injury: a control comparison. J Head Trauma Rehabil. juin 2013;28(3):171-8.

21. Simpson GK, Sabaz M, Daher M. Prevalence, clinical features, and correlates of inappropriate sexual behavior after traumatic brain injury: a multicenter study. J Head Trauma Rehabil. juin 2013;28(3):202-10.

22. Goldin Y, Cantor JB, Tsaousides T, Spielman L, Gordon WA. Sexual functioning and the effect of fatigue in traumatic brain injury. J Head Trauma Rehabil. oct 2014;29(5):418-26.

23. Yang $\mathrm{Y}-\mathrm{J}$, Chien $\mathrm{W}-\mathrm{C}$, Chung $\mathrm{C}-\mathrm{H}$, Hong K-T, Yu Y-L, Hueng D-Y, et al. Risk of Erectile Dysfunction After Traumatic Brain Injury: A Nationwide Population-Based Cohort study in Taiwan. Am J Mens Health. juill 2018;12(4):913-25.

24. Rosenbaum M, Najenson T. Changes in life patterns and symptoms of low mood as reported by wives of severly brain-injured soldiers. J Consult Clin Psychol. déc 1976;44(6):881-8.

25. Sabhesan S, Natarajan M. Sexual behavior after head injury in Indian men and women. Arch Sex Behav. août 1989;18(4):349-56.

26. Garden FH, Bontke CF, Hoffman M. Sexual functioning and marital adjustment after traumatic brain injury. J Head Trauma Rehabil. 1990;5(2):52-9.

27. O'Carroll RE, Woodrow J, Maroun F. Psychosexual and psychosocial sequelae of closed head injury. Brain Inj. sept 1991;5(3):303-13.

28. Gosling J, Oddy M. Rearranged marriages: marital relationships after head injury. Brain Inj. oct 1999;13(10):785-96.

29. Wedcliffe $T$, Ross $E$. The psychological effects of traumatic brain injury on the quality of life of a group of spouses/partners. South Afr J Commun Disord Suid-Afr Tydskr Vir Kommun. 2001;48:77-99.

30. Bivona U, Antonucci G, Contrada M, Rizza F, Leoni F, Zasler ND, et al. A biopsychosocial analysis of sexuality in adult males and their partners after severe traumatic brain injury. Brain Inj.

2016;30(9):1082-95. 
31. Sander AM, Maestas KL, Pappadis MR, Hammond FM, Hanks RA, Sander AM, et al. Multicenter Study of Sexual Functioning in Spouses/Partners of Persons With Traumatic Brain Injury. Arch Phys Med Rehabil. 1 mai 2016;97(5):753-9.

32. Downing M, Ponsford J. Sexuality in individuals with traumatic brain injury and their partners. Neuropsychol Rehabil. sept 2018;28(6):1028-37.

33. Hanks RA, Sander AM, Millis SR, Hammond FM, Maestas KL. Changes in sexual functioning from 6 to 12 months following traumatic brain injury: a prospective TBI model system multicenter study. J Head Trauma Rehabil. juin 2013;28(3):179-85.

34. Ponsford JL, Downing MG, Stolwyk R. Factors associated with sexuality following traumatic brain injury. J Head Trauma Rehabil. juin 2013;28(3):195-201.

35. Sander AM, Maestas KL, Nick TG, Pappadis MR, Hammond FM, Hanks RA, et al. Predictors of sexual functioning and satisfaction 1 year following traumatic brain injury: a TBI model systems multicenter study. J Head Trauma Rehabil. juin 2013;28(3):186-94.

36. Bellamkonda E, Zollman F. Relationship between employment status and sexual functioning after traumatic brain injury. Brain Inj. 2014;28(8):1063-9.

37. Moreno JA, McKerral M. Towards a taxonomy of sexuality following traumatic brain injury: A pilot exploratory study using cluster analysis. NeuroRehabilitation. 2017;41(2):281-91.

38. Moreno JA, McKerral M. Relationships between risky sexual behaviour, dysexecutive problems, and mental health in the years following interdisciplinary TBI rehabilitation. Neuropsychol Rehabil. janv 2018;28(1):34-56.

39. Zencius $A$, Wesolowski MD, Burke WH, Hough S. Managing hypersexual disorders in brain-injured clients. Brain Inj. juin 1990;4(2):175-81.

40. Simpson G, McCann B, Lowy M. Treatment of premature ejaculation after traumatic brain injury. Brain Inj. août 2003;17(8):723-9.

41. Simpson GK, McCann B, Lowy M. Treating male sexual dysfunction after traumatic brain injury: Two case reports. NeuroRehabilitation. 23 mars 2016;38(3):281-9.

42. Dolberg OT, Klag E, Gross Y, Schreiber S. Relief of serotonin selective reuptake inhibitor induced sexual dysfunction with low-dose mianserin in patients with traumatic brain injury.

Psychopharmacology (Berl). juin 2002;161(4):404-7.

43. Kelly G, Todd J, Simpson G, Kremer P, Martin C. The Overt Behaviour Scale (OBS): a tool for measuring challenging behaviours following $A B I$ in community settings. Brain Inj. mars 2006;20(3):307-19.

44. Gill CJ, Sander AM, Robins N, Mazzei DK, Struchen MA. Exploring experiences of intimacy from the viewpoint of individuals with traumatic brain injury and their partners. J Head Trauma Rehabil. févr 2011;26(1):56-68.

45. Lundberg PO, Ertekin C, Ghezzi A, Swash M, Vodusek D. Guidelines for Neurologists. 2001;23. 
46. Denys P, Soler J-M, Giuliano F. [Sexuality of men with neurologic disorders]. Progres En Urol J Assoc Francaise Urol Soc Francaise Urol. juill 2013;23(9):712-7.

47. Simpson G, Long E. An evaluation of sex education and information resources and their provision to adults with traumatic brain injury. J Head Trauma Rehabil. oct 2004;19(5):413-28.

48. Sander AM, Maestas KL. Information/education page. Sexuality after traumatic brain injury. Arch Phys Med Rehabil. sept 2014;95(9):1801-2.

49. Aloni R, Katz S. A review of the effect of traumatic brain injury on the human sexual response. Brain Inj. avr 1999;13(4):269-80.

50. Arango-Lasprilla JC, Olabarrieta-Landa L, Ertl MM, Stevens LF, Morlett-Paredes A, Andelic N, et al. Provider perceptions of the assessment and rehabilitation of sexual functioning after Traumatic Brain Injury. Brain Inj. 2017;31(12):1605-11.

51. Rees PM, Fowler CJ, Maas CP. Sexual function in men and women with neurological disorders. Lancet Lond Engl. 10 févr 2007;369(9560):512-25.

52. Poncet F, Pradat-DiehI P, Lamontagne M-E, Alifax A, Fradelizi P, Barette $M$, et al. Participant and service provider perceptions of an outpatient rehabilitation program for people with acquired brain injury. Ann Phys Rehabil Med. 1 sept 2017;60(5):334-40.

\section{LEGENDES DES FIGURES ET DES TABLEAUX}

Figure 1. Diagramme de sélection des articles

Tableau 1. Fréquence des dysfonctions sexuelles chez les patients traumatisés crâniens ou leur partenaire

Tableau 2. Facteurs associés aux dysfonctions sexuelles chez les patients traumatisés crâniens ou leur partenaire

Tableau 3. Outils utilisés pour l’évaluation des dysfonctions sexuelles chez les patients traumatisés crâniens ou leur partenaire

Tableau 4. Traitement des dysfonctions sexuelles chez le patient traumatisé crânien

\section{LIENS D'INTERETS}


Les auteurs déclarent ne pas avoir de liens d'intérêts en rapport avec cet article. 
Tableau 1. Fréquence des dysfonctions sexuelles chez les patients traumatisés crâniens ou leur partenaire

\begin{tabular}{|c|c|c|c|}
\hline Auteurs, année & $\begin{array}{l}\text { Design } \pm \text { durée } \\
\text { de suivi }\end{array}$ & Population & Fréquence des dysfonctions sexuelles \\
\hline \multicolumn{4}{|l|}{ Patients } \\
\hline $\begin{array}{l}\text { Kosteljanetz M et } \\
\text { al., } 1981 \text { (13) }\end{array}$ & $\begin{array}{l}\text { transversal, } \\
\text { mono-centrique }\end{array}$ & $\begin{array}{l}\mathrm{N}=19,100 \% \text { hommes, âge moyen : } 39 \text { ans. } \\
100 \% \text { de TC légers à } 47 \text { mois d'évolution }\end{array}$ & $58 \%$ rapportent au moins une DS : $53 \% \searrow$ désir, 42\% DE. \\
\hline $\begin{array}{l}\text { Kreutzer JS et al., } \\
1989(10)\end{array}$ & $\begin{array}{l}\text { transversal, } \\
\text { mono-centrique }\end{array}$ & $\begin{array}{l}\mathrm{N}=21,100 \% \text { hommes, } 76 \% \text { mariés, âge } \\
\text { moyen : } 39 \text { ans. } 57 \% \text { de TC sévères, à } 16,2 \\
\text { mois d'évolution }\end{array}$ & $\begin{array}{l}\text { Pas de prévalence globale des DS. } 57 \% \searrow \text { désir, } 14 \% \nearrow \text { désir, } 57 \% \\
D E, 62 \% \searrow \text { fréquence des RS, } 30 \% \text { dysorgasmie. }\end{array}$ \\
\hline $\begin{array}{l}\text { Sandel ME et al., } \\
1996(11)\end{array}$ & $\begin{array}{l}\text { transversal, } \\
\text { mono-centrique }\end{array}$ & $\begin{array}{l}\mathrm{N}=52,75 \% \text { hommes, } 33 \% \text { mariés ou en } \\
\text { couple, âge moyen : } 34,6 \text { ans. \% de TC } \\
\text { sévères inconnu, à 3,7 ans d'évolution }\end{array}$ & $\begin{array}{l}\text { Pas de prévalence globale des DS. Scores pathologiques } \\
\text { concernant l'orgasme et le désir. }\end{array}$ \\
\hline $\begin{array}{l}\text { Kreuter M et al., } \\
1998(12)\end{array}$ & $\begin{array}{l}\text { transversal, } \\
\text { mono-centrique }\end{array}$ & $\begin{array}{l}\mathrm{N}=92,70 \% \text { hommes, } 58 \% \text { mariés ou en } \\
\text { couple, âge moyen : } 40 \text { ans. } 97 \% \text { TC } \\
\text { sévères, à } 9 \text { ans d'évolution }\end{array}$ & $\begin{array}{l}\text { Pas de prévalence globale des DS. } 35 \% \searrow \text { désir, } 30 \% \\
\text { DE/éjaculation, } 40 \% \text { dysorgasmie, } 47 \% \searrow \text { fréquence des RS, } 26 \% \\
\text { n'ont plus eu de RS depuis le TC, } 19 \% \nearrow \text { masturbation. } 11 \% \text { des } \\
\text { patients en couples n'étaient pas satisfaits de la composante } \\
\text { sexuelle de leur relation. }\end{array}$ \\
\hline $\begin{array}{l}\text { Aloni A et al., } \\
1999(14)\end{array}$ & $\begin{array}{l}\text { transversal, } \\
\text { mono-centrique }\end{array}$ & $\begin{array}{l}\mathrm{N}=44,100 \% \text { hommes, dont } 64 \% \text { mariés ou } \\
\text { en couple, âge moyen : } 27 \text { ans. } \% \text { TC } \\
\text { sévères inconnu, à } 6 \text { semaines d'évolution }\end{array}$ & $\begin{array}{l}14 \% \text { rapportent au moins une DS. Patients célibataires : } 18 \% \\
\text { trouble du désir, } 12 \% \text { DE, } 59 \% \text { trouble de l'éjaculation et } 18 \% \\
\text { trouble de l'excitation. Patients en couple : } 4 \% \text { de DE et } 7 \% \text { de } \\
\text { dysorgasmie. }\end{array}$ \\
\hline $\begin{array}{l}\text { Crowe SF et al., } \\
1999(15)\end{array}$ & $\begin{array}{l}\text { étude cas-témoin, } \\
\text { mono-centrique }\end{array}$ & $\begin{array}{l}\mathrm{N}=28 \text { (dont } 14 \mathrm{TC} \text { ), } 100 \% \text { hommes, âge } \\
\text { moyen : } 32,4 \text { ans. } \% \text { TC sévères inconnu, à } \\
47 \text { mois d'évolution }\end{array}$ & $\begin{array}{l}100 \% \text { des patients rapportent au moins une DS (sélectionnés sur la } \\
\text { présence d'une DS) : } 59 \% \searrow \text { fonction sexuelle, } 86 \% \searrow \text { désir/ } \\
\text { fréquence RS, } 36 \% \text { dysorgasmie, } 43 \% \text { DE. Capacité à créer des } \\
\text { images visuelles à caractère sexuel significativement différente } \\
\text { des témoins. }\end{array}$ \\
\hline
\end{tabular}


Simpson $\mathrm{G}$ et al., 1999 (16)

cohorte

$\mathrm{N}=445,81.6 \%$ hommes, âge moyen : 30

rétrospective, ans. \% TC sévères inconnu

mono-centrique,

suivi 10 ans

Quintard B et al., 2002 (17)

transversal, multicentrique

$\mathrm{N}=79,75 \%$ hommes, $64 \%$ mariés ou en couple, âge moyen : 40,5 ans. 100 \% TC sévères, à 9,2 ans d'évolution

Ponsford J et al., 2003 (3)

étude cas-témoin, mono-centrique

$\mathrm{N}=358$ (dont $58 \%$ de TC). Cas : $69 \%$ hommes, $50 \%$ mariés ou en couple, âge moyen : 33,6 ans, $77 \%$ TC sévères, entre 1 5 ans d'évolution

Homme de 34 ans, TC dans l'enfance avec atrophie fronto-pariétale

Bianchi-Demiche
F et al., $2010(18)$

Sander AM et al., 2012 (19)

Downing MG et al., $2013(20)$

étude cas-témoin mono-centrique hommes, $32,4 \%$ mariés ou en couple, âge moyen : 34,7 ans, $72,3 \%$ TC sévères, à 3,5 ans d'évolution

Simpson GK et al., transversal, multi$2013(21)$ centrique
$\mathrm{N}=507,74 \%$ hommes, $26 \%$ mariés ou en couple, âge moyen : 37 ans. 100 \% TC sévères, à 2,2 ans d'évolution
6,5\% de l'échantillon ont commis une forme quelconque d'infraction sexuelle : frotteurisme> toucher le corps d'une personne (excluant les parties génitales) sans son consentement > exhibitionnisme> voyeurisme > agression sexuelle. Victimes : membres du personnel médical > public > autres patients avec un TC $>$ membres de la famille

$67 \%$ des patients sont insatisfaits de leur vie sexuelle

Pas de prévalence globale des DS : $54 \% \searrow$ fréquence RS, $41 \% \searrow$ désir/capacité à maintenir l'excitation, $39 \% \square$ capacité à satisfaire partenaire, 38\% \ capacité à initier un RS, 36\% dysorgasmie, $47 \%$ $\searrow$ sex appeal. Significativement différent des témoins

Développement d'une paraphilie attribuée aux séquelles de TC

Pas de prévalence globale des DS : $55 \% \searrow$ fréquence des RS, $15 \%$ altération fantasmes, $28 \%$ altération de l'excitation, $18 \%$ altération des expériences sexuelles et $34 \%$ dysorgasmie, 29,2\% sont insatisfaits sexuellement

Pas de prévalence globale des DS : $54,4 \% \searrow$ RS, $40,8 \% \searrow$ désir, $36,4 \% \searrow$ capacité à initier un RS, $37,8 \% \searrow$ capacité à satisfaire leur partenaire, $36,6 \%$ difficultés à maintenir l'excitation et 31,6\% dysorgasmie. Significativement différent des témoins

Prévalence des comportements sexuels inappropriés : 8,9\%. Paroles inappropriées $>$ toucher $>$ exhibitionnisme $>$ agression sexuelle 
Goldin $\mathrm{Y}$ et al. 2014 (22)

cas-témoin, mono-centrique

Strizzi J et al., 2015 (6)

Yang YJ et al., 2018 (2018)

cas-témoin, mono-centrique
$\mathrm{N}=283$ (dont $71 \%$ de TC). Patients : $52 \%$ hommes, $19 \%$ mariés ou en couple, âge moyen : 48 ans, \% TC sévères inconnu, à au moins 1 an d'évolution

$\mathrm{N}=58$ (dont $50 \%$ de TC). Patients : $0 \%$ hommes, $65,5 \%$ mariés ou en couple, âge moyen : 36,4 ans, \% TC sévères inconnu (tous modérés à sévères), à 18 mois d'évolution

cas-témoin niché $\quad \mathrm{N}=290514$ (dont 33\% de TC). Patients : dans une cohorte, $100 \%$ hommes, $65,5 \%$ mariés ou en couple, base de donnée de l'assurance maladie, suivi moyen 2,5 ans âge moyen : 36,4 ans, \% TC sévères inconnu (tous modérés à sévères), à 18 mois d'évolution
Pas de prévalence globale des DS. Absence de différence de fréquence de RS avec le groupe contrôle

$83 \%$ rapportent au moins une DS, non significativement différent des témoins. Différence significative avec $\searrow$ pour : désir, excitation, lubrification, orgasme et satisfaction sexuelle

Pas de prévalence globale des DS, données uniquement sur la DE.
A la fin du suivi, taux d'incidence de DE : 24,7/100000 dans le groupe TC vs 19,1/100000 dans le groupe témoin, significativement différent. Taux d'incidence cumulé sur 10 ans : $0,09 \%$ dans le groupe TC vs 0,07\%, significativement différent.

\section{Partenaires ou couples}

\section{Rosenbaum $\mathrm{M}$ et}

al., 1976 (24)

Sabhesan S et al., 1989 (25)

Garden FH et al., $1990(26)$

cas-témoin, mono-centrique

$\mathrm{N}=30$ épouses de vétérans (dont 33\% de TC), durée moyenne de mariage de 4,5 ans, âge moyen : 26 ans.

cohorte, monocentrique, durée suivi inconnue

$\mathrm{N}=34,82 \%$ hommes, $82 \%$ mariés ou en couple, âge moyen : 31,8 ans. \% de TC sévères inconnu, délai d'évolution inconnu

$\mathrm{N}=15$ couples, durée moyenne de mariage de 11,5 ans. Patients : $73 \%$ hommes, âge moyen : 39,2 ans. \% de TC sévères inconnu, à 11,4 mois d'évolution

O'Carroll RE et al., Transversal, 1991 (27) mono-centrique
$\mathrm{N}=36$ dont 17 couples, durée moyenne de mariage inconnu. Patients : $83 \%$ hommes, âge moyen : 35,6 ans. $39 \%$ de TC sévères, à 4,1 an d'évolution $\searrow$ des activités sexuelles et de l'importance d'être une partenaire sexuelle pour leur époux, $\nearrow$ des "difficultés" sexuelles de leur partenaire. Significativement différent du groupe témoin.

$62 \%$ rapportent au moins une DS : $14,7 \%$ comportement sexuel inapproprié, $23 \% \searrow$ fréquence des RS, trouble de l'excitation, ou éjaculation prématurée, $38 \%$ déclarent avoir retrouvé leur sexualité d'avant un an après le TC.

Couples : $47 \%$ sont insatisfaits, en rapport avec $\searrow$ fréquence des RS et désir. Patients : $36 \%$ DE ou de l'éjaculation, $50 \%$ de dysorgasmie. Partenaires : 25\% DE ou de l'éjaculation, $64 \%$ de dysorgasmie

$50 \%$ des patients et $9 \%$ des partenaires ont une DS 
Gosling J et al., 1999 (28)

Transversal, mono-centrique

Wedcliffe T et al., Transversal, 2001 (29) mono-centrique

Bivona $U$ et al., 2016 (30)

cas-témoin, mono-centrique

Sander AM et al. 2016 (31)

Downing $\mathrm{M}$ et al. 2018 (32)

cohorte, multicentrique, durée inconnue

transversal, mono-centrique
$\mathrm{N}=18$ couples hétérosexuels, durée moyenne de relation 16, 2 ans. Patients : $100 \%$ hommes, âge moyen : 42,1 ans, \% de TC sévères inconnu, à 4,1 an d'évolution $\mathrm{N}=14$ partenaires, durée moyenne du relation inconnue, $86 \%$ femmes, âge moyen : 39,3 ans. Patients : aucune information

$\mathrm{N}=40$ couples ( $50 \%$ de cas) hétérosexuels, durée moyenne de relation de 16 ans. Patients: $100 \%$ hommes, âge moyen : 42,1 ans, $100 \%$ TC sévères, à 2,5 ans d'évolution moyenne de suivi
$\mathrm{N}=70$ couples, durée moyenne de relation inconnue. Patients: $84 \%$ hommes, âge moyen : 45,1 ans, 62\% de TC sévères, à 1 an d'évolution

$\mathrm{N}=55$ couples, durée moyenne de relation inconnue. Patients: $74 \%$ hommes, âge moyen : 36,7 ans, $60 \%$ de TC sévères, à 3 ans d'évolution
Pas de prévalence globale des DS. Partenaires : 39\% עdésir chez le patient, $\searrow$ satisfaction sexuelle comparativement à la période avant le TC, 50\% ressentent les avances des patients comme coercitives au moins une partie du temps

Pas de prévalence globale des DS. $78,6 \%$ des couples n'ont plus de RS.

Pas de prévalence globale des DS \} \searrow \text { désir et fréquence des RS chez } les patients et partenaires, $\nearrow$ masturbation chez patients, comparativement aux témoins

$20 \%$ des partenaires rapportent une DS : $62 \% \searrow$ fréquence RS, $34 \% \searrow$ désir/importance sexualité, $44 \%$ insatisfaction sexuelle

$27,3 \%$ des patients ont une DS : 25,5\% trouble du désir, $21,8 \%$ trouble de l'excitation, 29,1\% trouble de l'orgasme

$16,4 \%$ des partenaires ont une DS : $20 \%$ trouble de l'excitation, $16,4 \%$ dysorgasmie ou trouble du désir. Différence significative uniquement sur trouble de la lubrification, plus fréquent chez les patientes que chez les partenaires 
Tableau 2. Facteurs associés aux dysfonctions sexuelles chez les patients traumatisés crâniens ou leur partenaire

\begin{tabular}{|c|c|c|c|}
\hline Auteurs, année & Facteurs sociodémographiques & $\begin{array}{l}\text { Facteurs liés au TC : sévérité, délai d'évolution, } \\
\text { localisation des lésions et séquelles }\end{array}$ & $\begin{array}{c}\text { Symptômes } \\
\text { neuropsychiatriques }\end{array}$ \\
\hline $\begin{array}{l}\text { Kosteljanetz M et } \\
\text { al., } 1981 \text { (13) }\end{array}$ & & $\begin{array}{l}\text { Association entre troubles cognitifs sévères et DS } \\
(p<0,05)\end{array}$ & \\
\hline $\begin{array}{l}\text { Sabhesan S et al., } \\
1989(25)\end{array}$ & $\begin{array}{l}\text { Sexe masculin associé aux DS }(p<0,05) \text {. } \\
\text { Pas d'association de l'âge ou du niveau } \\
\text { d'éducation avec les DS }\end{array}$ & Absence d'association entre sévérité du TC et DS & $\begin{array}{l}\text { Association entre "troubles } \\
\text { psychiatriques tardifs" et DS } \\
(p<0,01) \text {. }\end{array}$ \\
\hline $\begin{array}{l}\text { Kreutzer JS et al., } \\
1989(10)\end{array}$ & & & $\begin{array}{l}\text { Absence de corrélation entre } \\
\text { troubles de l'humeur et DS }\end{array}$ \\
\hline $\begin{array}{l}\text { O'Carroll RE et al., } \\
1991(27)\end{array}$ & $\begin{array}{l}\text { Corrélation positive entre âge et DS } \\
\text { chez les patients }(p=0,016)\end{array}$ & $\begin{array}{l}\text { Corrélation négative entre délai d'évolution du TC et } \\
\text { éjaculation prématurée }(p=0,019) \text { ou évitement des RS } \\
(p=0,030) \text { chez les patients. Corrélation positive entre } \\
\text { délai d'évolution du TC et insatisfaction sexuelle } \\
(p=0,012) \text { chez les patients. Absence de corrélation entre } \\
\text { sévérité du TC et DS chez le patient ou son partenaire. }\end{array}$ & $\begin{array}{l}\text { Corrélation positive entre score } \\
\text { HAD dépression et } D S(p=0,038) \\
\text { chez les patients et les } \\
\text { partenaires femmes ( } p=0,006) \text {. } \\
\text { Chez les partenaires femmes, } \\
\text { corrélation positive entre score } \\
\text { HAD Anxiété et } D S(p=0,001)\end{array}$ \\
\hline
\end{tabular}

Sandel ME et al., 1996 (11)
Corrélation négative entre délai d'évolution du TC et excitation sexuelle $(p=0039)$. Absence de corrélation entre DS et sévérité du TC (mesuré par la durée d'APT, le GCS ou l'échelle de Russell modifiée) ou fonction cognitive. Pas d'association entre localisation des lésions et $D S$. Atteinte du lobe frontal : $\nearrow$ fantasmes ou pensées liées au sexe $(p=0,04)$. Atteinte hémisphérique droite : $\lambda$ excitation $(p=0,044)$ et expériences sexuelles $(p=$ 0,026) 
Kreuter $\mathrm{M}$ et al. 1998 (12)

Simpson G et al., $1999(16)$

Quintard B et al., 2002 (17)

Ponsford J 2003 (3)

Sander AM et al., 2012 (19)

Hanks RA et al.,

2013 (même

cohorte que Sander

et al 2012) (33)

Downing MG et al., $2013(20)$

Ponsford JL et al., 2013 (même

cohorte que

Downing et a

2013) (34) moment du TC $(p<0,01)$ ou mauvais état de santé perçu $(p<0,001)$ et DS.

L'état de santé physique perçu est un

facteur indépendant de DS

Association négative entre âge au moment du TC et risque d'infraction sexuelle $(p<0,001)$

\section{Corrélation positive entre âge et DS ( $p$ $=0,002)$. Pas de corrélation avec le sexe. \\ Association entre sexe féminin et DS concernant les fantasmes $(p<0,05)$ et l'excitation $(p<0,01)$ \\ Association entre âge et sexe masculin à l'insatisfaction sexuelle}

Association entre âge entre 46-55 ans et DS $(p<0,001)$

Association positive entre âge et DS $(\mathbf{p}<\mathbf{0 , 0 0 1 )}$. Pas d'association avec le sexe
Association positive entre sévérité du TC mesurée par la GOS (mais pas par la durée d'APT) et DS ( $p<0,001$ ). Absence d'association entre handicap mesuré par la MIF et $D S$

Association positive entre sévérité du TC et risque d'infraction sexuelle $(p<0,001)$

\section{Association entre score faible à la GOS et insatisfaction} sexuelle

Absence de corrélation entre DS et délai d'évolution ou sévérité du TC mesurée par la durée d'APT

Corrélation entre score à l'échelle HADS pour l'anxiété ( $p$ $<.0001)$, pour la dépression $(p<$ $.0001)$ et DS

Amélioration de l'excitation à 1 an comparativement à 6 mois d'évolution du TC $(p<0,05)$

Association entre perte autonomie et DS $(p<0,05)$. Association entre dépression et Association négative avec le délai d'évolution $(p<0,05)$. DS $(p<0,001)$. Association Pas d'association entre sévérité du TC mesurée par la négative entre estime de soi et durée d'APT et DS DS $(p<0,001)$ 
Sander AM et al., 2013 (même

cohorte que Sander

et al 2012) (19)

Simpson GK et al., $2013(21)$

Bellamkonda $\mathrm{E}$ et

al., 2014 (36)

Goldin Y et al., 2014 (22)

Strizzi J et al., 2015 2016 (30)
Association entre DS et : âge $(p<0,0001)$, sexe féminin $(p=0,01)$ et participation sociale $(p=0,0009)$

Association négative entre âge au moment du TC $(p<0,05)$

comportement sexuel inapproprié

Association entre "bas" niveau de revenu (<40 000\$/an) et trouble de l'orgasme/éjaculation $(p=0,003)$ ou trouble du désir $(p=0,01)$. Absence de différence pour les catégories employé, chômage ou étudiant.

Association négative entre sexe féminin et fréquence $(p=0,006)$ et importance $(p=0,01)$ des RS. Association négative entre âge et importance des RS ( $p=0,008)$

Association positive entre âge et Association négative entre délai d'évolution et trouble trouble de l'excitation sexuelle $(p=$ de l'excitation sexuelle $(p=0,006)$, trouble de la $0,006)$, trouble de la lubrification $(p=$ lubrification $(p=0,018)$, dysorgasmie $(p=0,001)$. 0,005), dysorgasmie $(p=0,018)$ et Absence d'association entre sévérité du TC évaluée par le trouble du désir $(p<0,001)$

GCS et trouble de l'excitation sexuelle, trouble de la lubrification, dysorgasmie ou trouble du désir en analyse multivariée. Absence d'association entre délai d'évolution et trouble du désir.

Pas de corrélation entre âge ou niveau d'éducation et fréquence des RS
Association négative entre fatigue et fréquence des RS ( $p=$ 0,04)

Pas de corrélation entre fréquence des RS et anxiété ou dépression 
Sander AM et al., 2016 (31)

Moreno JA et al., 2017 (37)

Moreno JA et al., 2018 (38)

Yang YJ et al., 2018 (23)

Fonctionnement sexuel du patient
$(p<0,01)$ et âge du partenaire $(p<0,01)$ associé aux DS chez le partenaire

Absence d'association entre DS chez le partenaire et

Association positive entre âge et DS $(p=0,00001)$ gravité du TC mesuré par la durée d'APT, la durée de

\section{participation sociale}

\section{Association positive entre DS et anxiété généralisée \\ $(p=0,00004)$ ou dépression $(p=0,00001)$}

Corrélation positive entre comportement sexuel à risque et trouble des fonctions exécutives $(p<0,05)$. Absence de corrélation entre comportement sexuel à risque et Absence de corrélation entre comportement sexuel à risque et anxiété ou dépression perte de conscience ou le GCS.

Association entre sévérité du TC et dysfonction érectile (p $<0,001)$

En gras : les associations significatives en analyse multivariée ; en italique : les associations non significatives ; APT : amnésie post-traumatique ; DS :

dysfonction sexuelle ; GCS : Glasgow coma scale ; GOS : Glasgow outcome scale ; TC : traumatisme crânien 
Tableau 3. Outils utilisés pour l'évaluation des dysfonctions sexuelles chez les patients traumatisés crâniens ou leur partenaire

\begin{tabular}{|c|c|c|c|}
\hline Nom de l'outil & $\begin{array}{l}\text { Etudes } \\
\text { utilisant l'outil }\end{array}$ & $\begin{array}{l}\text { Outil validé pour } \\
\text { l'évaluation des } \\
\text { DS }\end{array}$ & $\begin{array}{l}\text { Validation dans une } \\
\text { population de TC / } \\
\text { neurologique }\end{array}$ \\
\hline $\begin{array}{l}\text { American Medical Association's Self- } \\
\text { Evaluation of Sexual Behavior and } \\
\text { Gratification (AMAS-ESBG) }\end{array}$ & (26) & \multicolumn{2}{|c|}{ Outil non retrouvé } \\
\hline $\begin{array}{l}\text { Brain Injury Questionnary of Sexuality } \\
\text { (BIQS) }\end{array}$ & $(20,31,34)$ & Oui & $\begin{array}{l}\text { Oui par Stolwyk RJ et } \\
\text { al., } 2013\end{array}$ \\
\hline $\begin{array}{l}\text { Derogatis Interview for Sexual } \\
\text { Function (DISF) }\end{array}$ & $\begin{array}{l}(11,19,31,33,3 \\
5,36)\end{array}$ & Oui & Non/ Non \\
\hline $\begin{array}{l}\text { Derogatis Interview for Sexual } \\
\text { Function- Self Reported (DISF-SR) }\end{array}$ & $\begin{array}{l}(19,31- \\
33,35,36)\end{array}$ & Oui & Non / Non \\
\hline $\begin{array}{l}\text { Female Sexual Functioning Index } \\
\text { (FSFI) }\end{array}$ & (6) & Oui & Non/Non \\
\hline $\begin{array}{l}\text { Life satisfaction questionnaire, item } \\
\text { satisfaction vie sexuelle }\end{array}$ & $(17)$ & Non & Non/non \\
\hline $\begin{array}{l}\text { Golombok Rust Inventory of Sexual } \\
\text { Satisfaction (GRISS) }\end{array}$ & $(27,28)$ & Oui & Non/non \\
\hline Index of Sexual Satisfaction (ISS) & (6) & Oui & Non/ Non \\
\hline Overt Behavior Scale (OBS) & $(21)$ & Oui & $\begin{array}{l}\text { Oui par Kelly G et al., } \\
2016\end{array}$ \\
\hline $\begin{array}{l}\text { Psychosexual Assessment } \\
\text { Questionnaire (PAQ) }\end{array}$ & $(10,14,15,20)$ & Non & $\begin{array}{l}\text { Non, mais création } \\
\text { dans une population } \\
\text { de TC par Kreutzer JS } \\
\text { et al., } 1989\end{array}$ \\
\hline $\begin{array}{l}\text { Sexual adjustement Questionnaire } \\
\text { (SAQ) }\end{array}$ & $(12)$ & Oui & $\begin{array}{l}\text { Non / Oui, population } \\
\text { de médullo-lésés }\end{array}$ \\
\hline Sexual Desire Inventory (SDI-2) & (6) & Oui & Non / Non \\
\hline Sexual Interest and Satisfaction (SIS) & $(12)$ & Oui & $\begin{array}{l}\text { Non/ Oui, population } \\
\text { de médullo-lésés }\end{array}$ \\
\hline $\begin{array}{l}\text { Sexual Quality of Life Questionnaire } \\
\text { (SQoL) }\end{array}$ & (6) & Oui & Non / Non \\
\hline $\begin{array}{l}\text { Sexuality Evaluation Schedule } \\
\text { Assesment Monitoring (SESAMO) }\end{array}$ & (30) & Oui & Non / Non \\
\hline $\begin{array}{l}\text { Vivdness of sexual imagery scale of } \\
\text { the imaginal processes inventory } \\
\text { (VSISIPI) }\end{array}$ & $(15)$ & Non & Non/Non \\
\hline
\end{tabular}


Tableau 4. Traitement des dysfonctions sexuelles chez le patient traumatisé crânien

\begin{tabular}{|c|c|c|c|c|}
\hline $\begin{array}{l}\text { Auteurs, } \\
\text { année }\end{array}$ & Design & $\begin{array}{c}\text { Population et type } \\
\text { de DS }\end{array}$ & $\begin{array}{c}\text { Type de prise en } \\
\text { charge }\end{array}$ & $\begin{array}{c}\text { Efficacité et } \\
\text { tolérance }\end{array}$ \\
\hline $\begin{array}{l}\text { Zencius A et } \\
\text { al., } 1990 \text { (39) }\end{array}$ & Série de cas & $\begin{array}{l}\text { Comportement } \\
\text { sexuel inapproprié } \\
\text { chez } 3 \text { patients }\end{array}$ & $\begin{array}{l}\text { Psycho- } \\
\text { comportementale }\end{array}$ & $\begin{array}{l}\text { Absence } \\
\text { d'évaluation } \\
\text { statistique, } \\
\text { amélioration } \\
\text { clinique dans les } \\
3 \text { cas }\end{array}$ \\
\hline $\begin{array}{l}\text { Dolberg OT et } \\
\text { al., } 2002 \text { (42) }\end{array}$ & $\begin{array}{l}\text { Etude non } \\
\text { randomisée }\end{array}$ & 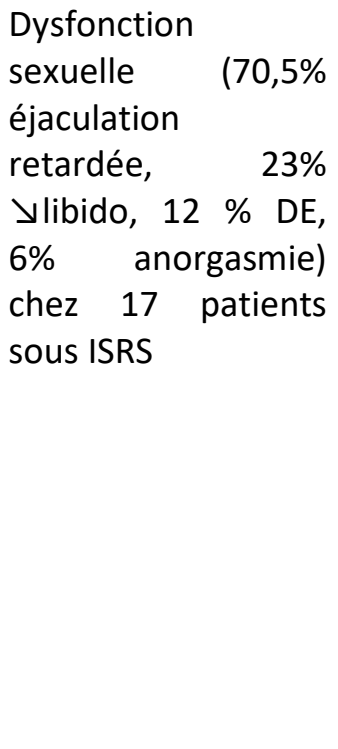 & $\begin{array}{l}\text { Ajout de Miansérine } \\
\text { de } 7,5 \text { à } 15 \mathrm{mg} / \text { jour }\end{array}$ & $\begin{array}{l}\text { Absence } \\
\text { d'évaluation } \\
\text { statistique, } 88 \% \\
\text { d'amélioration à } \\
2 \text { mois, dont } \\
59 \% \text { (toutes les } \\
\text { éjaculations } \\
\text { retardées) avec } \\
\text { une disparition } \\
\text { des symptômes } \\
\text { de DS. Effets } \\
\text { secondaires } \\
\text { dans 35\% des } \\
\text { cas : vertiges, } \\
\text { sécheresse } \\
\text { buccale }\end{array}$ \\
\hline $\begin{array}{l}\text { Simpson G et } \\
\text { al., } 2003(40)\end{array}$ & Cas clinique & $\begin{array}{l}\text { Ejaculation } \\
\text { prématurée chez } 1 \\
\text { patient }\end{array}$ & $\begin{array}{l}\text { Psycho- } \\
\text { comportementale } \\
\text { (sexologue), } \\
\text { anesthésique locale }\end{array}$ & Amélioration \\
\hline $\begin{array}{l}\text { Simpson GK et } \\
\text { al., } 2016 \text { (41) }\end{array}$ & Série de cas & $\begin{array}{l}\text { Ejaculation retardée } \\
(1 \quad \text { cas }) \quad \text { et } \\
\text { dysfonction érectile } \\
\text { (1 cas) }\end{array}$ & $\begin{array}{l}\text { Psycho- } \\
\text { comportementale } \\
\text { (sexologue), } \\
\text { médicamenteuse }\end{array}$ & $\begin{array}{l}\text { Absence } \\
\text { d'évaluation } \\
\text { statistique, } \\
\text { amélioration } \\
\text { clinique dans les } \\
2 \text { cas }\end{array}$ \\
\hline
\end{tabular}

DE : dysfonction érectile ; DS : dysfonction sexuelle ; ISRS : inhibiteur sélectif de recapture de la sérotonine ; TC : traumatisme crânien 